\title{
Propulsion Technology Assessment: Science \& Enabling Technologies to Explore the Interstellar Medium
}

\author{
Randall C. Hopkins ${ }^{1}$, Herbert D. Thomas ${ }^{1}$, Bruce M. Wiegmann ${ }^{1}$, Andrew F. Heaton ${ }^{2}$ and Les Johnson ${ }^{3}$ \\ NASA, George C. Marshall Space Flight Center, AL, 35812, United States \\ Michael F. Baysinger ${ }^{1}$ \\ Jacobs ESSSA Group, Huntsville, AL 35806, United States \\ Benjamin R. Beers ${ }^{4}$ \\ Geocent, LLC. - Jacobs ESSSA Group, Huntsville, AL, 35806, United States
}

\begin{abstract}
As part of a larger effort led by the Keck Institute for Space Studies at the California Institute of Technology, the Advanced Concepts Office at NASA's George C. Marshall Space Flight Center conducted a study to assess what low-thrust advanced propulsion system candidates, existing and near term, could deliver a small, Voyager-like satellite to our solar system's heliopause, approximately $100 \mathrm{AU}$ from the center of the sun, within 10 years and within a 2025 to 2035 launch window. The advanced propulsion system trade study consisted of three candidates, including a Magnetically Shielded Miniature (MaSMi) Hall thruster, a solar sail and an electric sail. Two aerial densities, and thus characteristic accelerations, $0.426 \mathrm{~mm} / \mathrm{s}^{2}$ and $0.664 \mathrm{~mm} / \mathrm{s}^{2}$, were analyzed for the solar sail option in order understand the impact of near and long term development of this technology. Similarly, two characteristic accelerations, $1 \mathrm{~mm} / \mathrm{s}^{2}$ and $2 \mathrm{~mm} / \mathrm{s}^{2}$, were also analyzed for the electric sail option in addition to tether quantities of 10 and 20, respectively, and individual tether length of $20 \mathrm{~km}$. A second analysis was conducted to determine what existing solid rocket motor kick stage(s) would be required to provide additional thrust at various points in the trajectory, assuming an earth departure characteristic energy capability provided by a Space Launch System (SLS) Block 1B vehicle architecture carrying an 8.4 meter payload fairing. Two trajectory profiles were considered, including an escape trajectory using a Jupiter gravity assist $(E-J u)$, and an escape trajectory first performing a Jupiter gravity assist followed by an Oberth maneuver around the sun and an optional Saturn gravity assist $(E-J u-S u-S a)$. The Oberth maneuver would need to be performed very close to the sun, wherein this study assumed a perihelion distance of approximately 11 solar radii, or $0.05 \mathrm{AU}$, away from the surface. The heat shield technology required to perform this type of ambitious maneuver was assumed to be similar to that of NASA's Solar Probe Plus mission, which is slated to launch in July 2018. With respect to a SLS Block 1B earth departure characteristic energy capability of $100 \mathrm{~km}^{2} / \mathrm{s}^{2}$ for the $E-J u$ trajectory option, results indicated that compared to having no advanced propulsion system onboard, both the MaSMi Hall thruster and solar sail options subtract approximately 8 to 10 years from the total trip time while the electric sail outperforms all options by subtracting up to 20 years. With respect to an average kick stage velocity capability of 2.5 to $3.5 \mathrm{~km} / \mathrm{s}$ at perihelion, the most sensitive segment of the $\mathrm{E}-\mathrm{Ju}-\mathrm{Su}-\mathrm{Sa}$ trajectory option, results indicated that both the MaSMi Hall thrust and solar sail options only subtract 1 to 3 years from the total trip time whereas the electric sail again outperforms all other options by subtracting up to 5 years. In other words, if the Technology Readiness Level of an electric sail could be increased in time, this propulsion technology could not only enable a satellite to reach $100 \mathrm{AU}$ in 10 years but it could potentially do so even faster. Completing such an ambitious mission in that short of a timespan would be very attractive to many as it would be well within the average career span of any of those involved.
\end{abstract}

\footnotetext{
${ }^{1}$ Aerospace Engineer, Advanced Concepts Office, NASA/MSFC ED04

${ }^{2}$ Aerospace Engineer, Guidance, Navigation and Mission Analysis, NASA/MSFC EV42, AIAA Professional Member

${ }^{3}$ Technical Assistant, Advanced Concepts Office, NASA/MSFC ED04

${ }^{4}$ Aerospace Engineer, Advanced Concepts Office, NASA/MSFC ED04, AIAA Professional Member
} 


\section{Introduction}

If you look up into the sky on an especially clear night, it is likely you will become transfixed on the stars above. Whether you are waiting to see a shooting star to pass overhead, or you are gazing into the abyss simply wondering about what is above, that moment where you find yourself pondering the universe is something that has captivated man for quite some time. It is a natural reaction for man to desire to understand the world, and in this case the cosmos, in which he lives; to understand something inherently requires knowledge of it.

The Voyager Interstellar Mission (VIM) was NASA's first attempt at acquiring in situ knowledge of the distant solar system, our stepping stone to the rest of the universe. Twin spacecraft, Voyager 1 and Voyager 2, after touring most of our solar system's outer planets, headed on their way toward interstellar space. The VIM was designed to collect data on three distinct phenomena: (1) termination shock, "where the solar winds slow from supersonic to subsonic speed and large changes in plasma flow direction and magnetic field orientation occur"; (2) heliosheath environment, "which is [an environment] still dominated by the Sun's magnetic fields and particles contained in the solar wind"; and (3) interstellar medium, "where the Sun's influence wanes and the beginning of interstellar space can be sensed." ${ }^{1}$ Launched in late summer of 1977, Voyager 1 took approximately 27 years just to reach the solar system's termination shock, the first of the three major phenomena at 94 Astronomical Units (AU) from the sun, and 36 years to reach the Interstellar Medium (ISM) with Voyager 2 not far behind. ${ }^{2}$ Although much data has been collected thus far, 36 years is essentially the entire span of one's career. And with the Voyager spacecraft expecting to shutdown sometime soon after the year 2020, ${ }^{1}$ it is time for NASA to dawn the next interstellar spacecraft that will augment our current knowledge of our solar system and the universe.

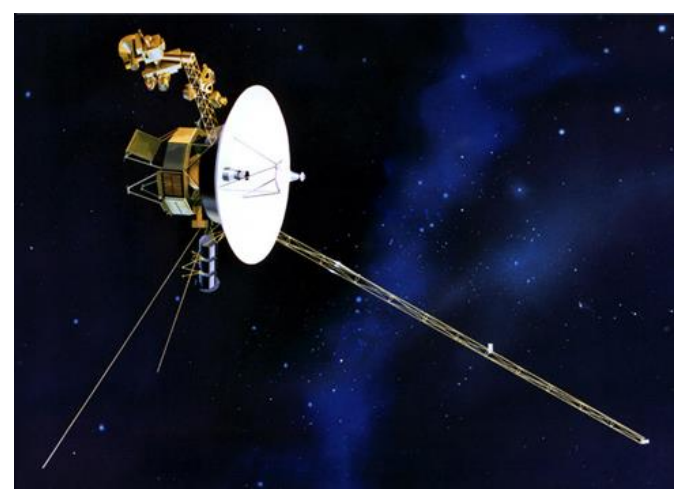

Figure 1. Artistic rendering of one of the twin Voyager spacecraft. (Image credit: NASA JPL)

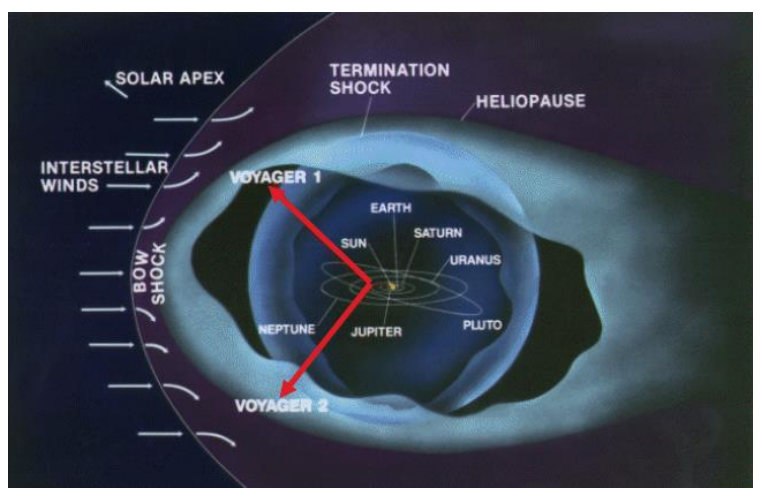

Figure 2. Voyager spacecraft trajectories within cosmic phenomena. (Image credit: NASA JPL)

Leveraging the success of the VIM, the Keck Institute for Space Studies (KISS) at the California Institute of Technology is studying Science and Enabling Technologies to Explore Interstellar Medium and is extending the planning for a potential mission to go there known as the Interstellar Probe. Although there will certainly be some science conducted throughout its journey, in comparison to the VIM, Table 1 below describes the overarching goals of the Interstellar Probe mission.

Table 1. Comparison of overarching goals for the Interstellar Probe mission. ${ }^{3}$

\begin{tabular}{|cl|l|l|}
\hline & Goals & \multicolumn{1}{|c|}{ Voyager } & \multicolumn{1}{c|}{ Interstellar Probe } \\
\hline 1. & Get there sooner & 100 AU in 29 years & 100+ AU in 10 years \\
\hline 2. & Travel Faster & approx. 3.6 AU/year & 18-36 AU/year \\
\hline 3. & Survive longer & approx. 43 years & 50-100 years \\
\hline
\end{tabular}

One of the biggest hurdles to overcome is designing and implementing a low-thrust advanced propulsion system (APS) which can accomplish the speedier travel and sooner arrival time to our solar system's termination shock, the beginning of the heliopause, at approximately $100 \mathrm{AU}$. The role of the NASA Marshall Space Flight Center's (MSFC) Advanced Concepts Office (ACO) was to conduct a trade study comparing known or near term low-thrust APS candidates while determining which Space Launch System (SLS) vehicle configuration could deliver the most appropriate characteristic energy $\left(\mathrm{C}_{3}\right)$ to the spacecraft based on several viable trajectory options. The candidate low- 
thrust APSs considered included a Magnetically Shielded Miniature (MaSMi) Hall thruster (Fig. 3), solar sails (Fig. 4) and electric sails (E-Sails) (Fig. 5). Several possible trajectories were studied involving both single- and multiple-planetary flyby maneuvers to understand if any additional "free" energy could be obtained to boost the speed of the spacecraft toward interstellar space. Further insight into each of these topics is included in subsequent sections of this paper.

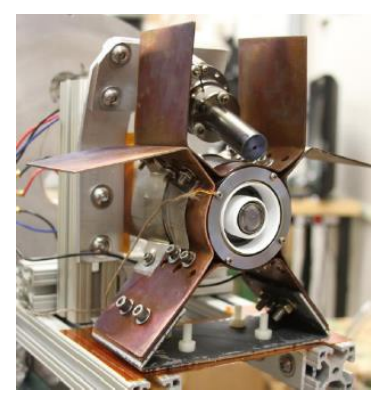

Figure 3. MaSMi Hall thruster system. (Image credit: UCLA)

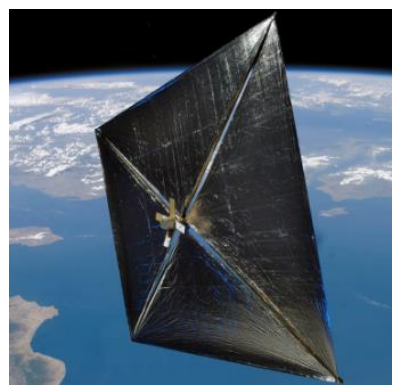

Figure 4. NanoSail-D solar sail system. (Image credit: NASA Science News)

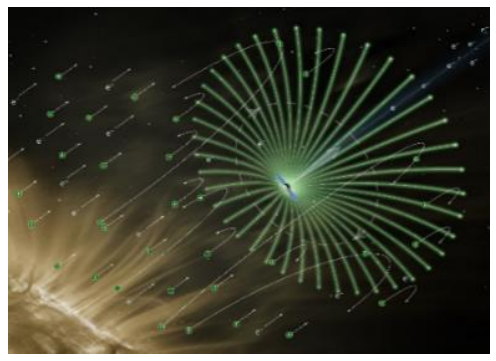

Figure 5. Electric sail (E-Sail) system. (Image credit: Szames)

\section{Background}

Earlier in FY2014, an initial Interstellar Probe study was also conducted through ACO, which targeted a design solution for a heavier spacecraft closer to that of Voyager 1 and 2, to reach $250 \mathrm{AU}$ in approximately 30 years. The goal was to generate a rough estimate of the possible performance employing only high-thrust chemical propulsion stages while launching on SLS. Table 2 describes some of the high-level ground rules and assumptions (GR\&A) applied.

Table 2. Ground Rules \& Assumptions (GR\&A) for initial Interstellar Probe study.

\begin{tabular}{|l|l|}
\hline \multicolumn{1}{|c|}{ Item } & \multicolumn{1}{c|}{ Description } \\
\hline Mission Performance & $250 \mathrm{AU}$ in 30 years \\
\hline Launch Window & $2025 \pm 2$ years \\
\hline Launch Vehicle & SLS Block 1B + EUS + 5.0 m PLF \\
\hline Spacecraft Mass & $725 \mathrm{~kg}\left(1,598 \mathrm{lb}_{\mathrm{m}}\right)$ \\
\hline Spacecraft Volume & Volume of Voyager (stowed) \\
\hline
\end{tabular}

The SLS is NASA's next generation super heavy lift launch vehicle system, following the retirement of the Space Transportation System (STS) in 2011, allowing NASA to continue its pursuit of human and non-human exploration endeavors. Throughout the life of the program the SLS will undergo two major block upgrades from its Block 1 baseline architecture following the B path upgrade scheme. The B path is comprised of first upgrading to Block 1B per the larger Exploration Upper Stage (EUS) from the Interim Cryogenic Propulsion Stage (iCPS) and then upgrading its two STS-era solid rocket motors (SRMs) to either a more powerful pair of advanced SRMs or liquid rocket boosters (LRBs) for Block 2B. So, the initial Interstellar Probe study assumed a SLS Block 1B vehicle architecture with a $5.0 \times 19.1 \mathrm{~m}(16.4 \times 62.7 \mathrm{ft})$ payload fairing $(\mathrm{PLF})$ based on its projected characteristic energy $\left(\mathrm{C}_{3}\right)$ capabilities (Fig. 6). ( $\mathrm{A} \mathrm{C}_{3}$ curve for the SLS Block 1B architecture with a $5.0 \mathrm{~m}$ (16.4 ft) PLF was not included in this report because the data will not be officially released in the SLS Program Mission Planner's Guide (MPG) Executive Overview until the February 2015 timeframe). ${ }^{4}$

The initial study investigated three potential mission profiles, each one targeting a 250 AU spacecraft delivery point based on the aforementioned launch vehicle architecture selection: (1) a direct escape trajectory per SLS Block $1 \mathrm{~B} \mathrm{C}_{3}$ performance capability, (2) an escape trajectory using a powered or unpowered Jupiter Gravity Assist (JGA), and (3) an escape trajectory using powered or unpowered Saturn-Uranus gravity assists. Lastly, the spacecraft mass was adjusted to determine how much it might impact the overall mission timeline. 


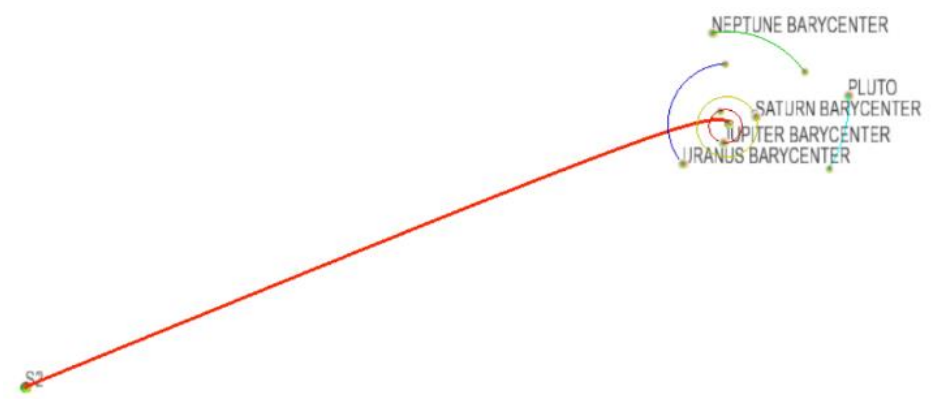

Figure 6. Direct insertion trajectory profile to $250 \mathrm{AU}$ without gravity assists.

The first mission profile employed no additional velocity $(\Delta \mathrm{V})$ gains except that which could be imparted strictly by the SLS Block 1B architecture with a $5.0 \mathrm{~m}$ (16.4 ft) PLF (Fig. 7). The mission profile was an Earth escape trajectory directly to an orbit with aphelion of $250 \mathrm{AU}$, neglecting the goal of reaching that distance in 30 years.

The required heliocentric velocity at earth departure of $42.0 \mathrm{~km} / \mathrm{s}(137,800 \mathrm{ft} / \mathrm{s})$ translates to a $\mathrm{C}_{3}$ energy of about $150 \mathrm{~km}^{2} / \mathrm{s}^{2}$, approximately $40 \mathrm{~km}^{2} / \mathrm{s}^{2}$ greater than the upper bound of the projected SLS Block $1 \mathrm{~B} \mathrm{C}_{3}$ capability for the assumed spacecraft mass. Therefore, the spacecraft would not be able to even reach 250 AU without employing either a kick stage or gravity assist, let alone be able to reach that distance in 30 years. Using a kick stage at earth departure, however, greatly reduces the total trip time. To estimate the optimal split between kick stage mass and SLS payload capability, various kick stage Propellant Mass Fractions (PMF) were assumed where Fig. 8 shows

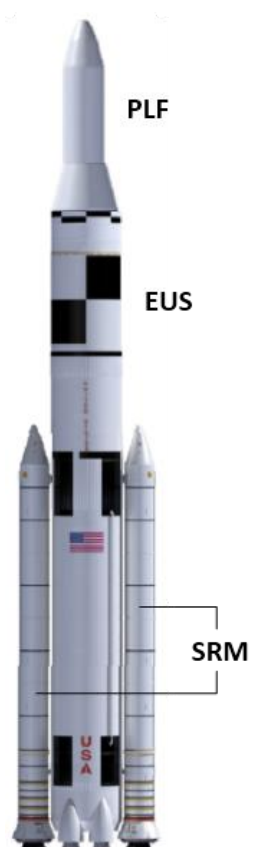

Figure 7. SLS Block 1B with EUS and $5.0 \mathrm{~m}$ PLF (adapted). (Image credit: The Boeing Company) the relationship between spacecraft velocity after kick stage burnout and $\mathrm{SLS} \mathrm{C}_{3}$ values. A theoretical kick stage with a PMF of 0.90 and specific impulse $\left(\mathrm{I}_{\mathrm{sp}}\right)$ of 450 seconds (approximately equivalent to a common Centaur) could get the spacecraft to $250 \mathrm{AU}$ in about 68 years assuming an SLS C $\mathrm{C}_{3}$ capability of $67.8 \mathrm{~km}^{2} / \mathrm{s}^{2}$.

The second mission profile incorporated a JGA (Fig. 10) and either the same scalable earth departure kick stage as shown above or a SRM. Setting the flyby radius at $350,000 \mathrm{~km}(217,490 \mathrm{mi})$ resulted in a trip time of about 49 years to $250 \mathrm{AU}$ for an unpowered flyby. Lowering the flyby distance to $126,000 \mathrm{~km}(78,296 \mathrm{mi})$ reduced the time by only a couple of years, still well short of the goal. Adding an additional kick stage for a powered flyby reduces the available mass for the earth departure kick stage and thus departure energy. Figure 9 describes several cases that were analyzed, including $1 \mathrm{~km} / \mathrm{s}, 2 \mathrm{~km} / \mathrm{s}, 3 \mathrm{~km} / \mathrm{s}$ and $4 \mathrm{~km} / \mathrm{s}$. In particular, assuming a SRM kick stage with an $\mathrm{I}_{\mathrm{sp}}$ of 292 seconds and PMF of 0.90 for the flyby maneuver indicated that a Star 63D motor fit well for a $4 \mathrm{~km} / \mathrm{s}$ powered flyby. But for this configuration, a Star 63F SRM provided the best performance for an earth departure stage resulting in a total trip time of 40.5 years, while all other cases yielded a longer trip time.

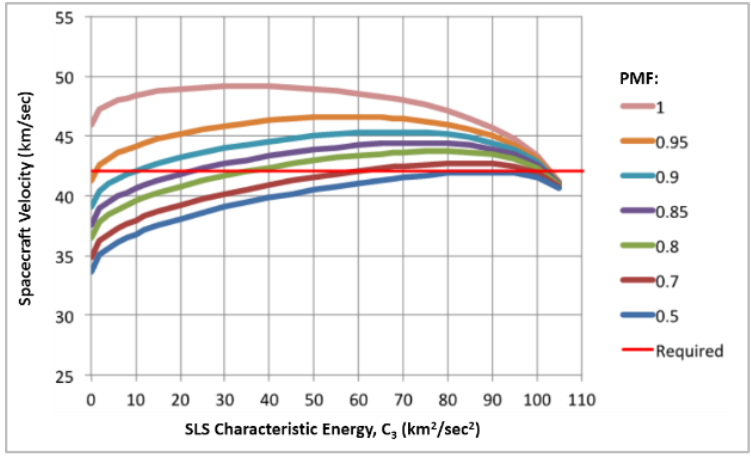

Figure 8. Propellant Mass Fraction (PMF) sweep to size the spacecraft kick stage assuming 450 seconds of $\mathbf{I}_{\text {sp. }}$.

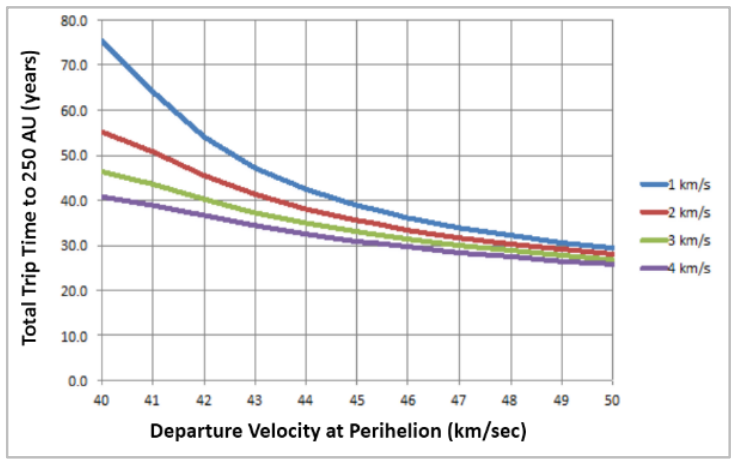

Figure 9. Various trip times to 250 AU for a powered Jupiter Gravity Assist (JGA) trajectory. 


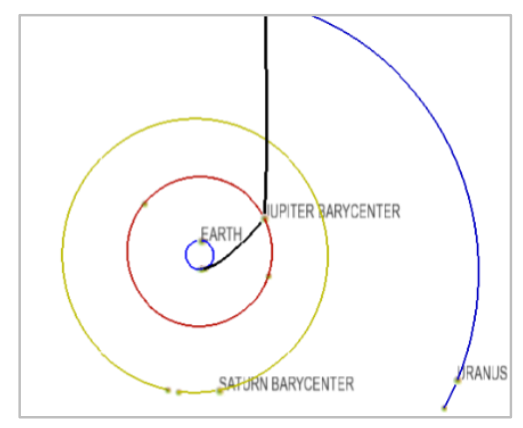

Figure 10. Powered JGA trajectory profile.

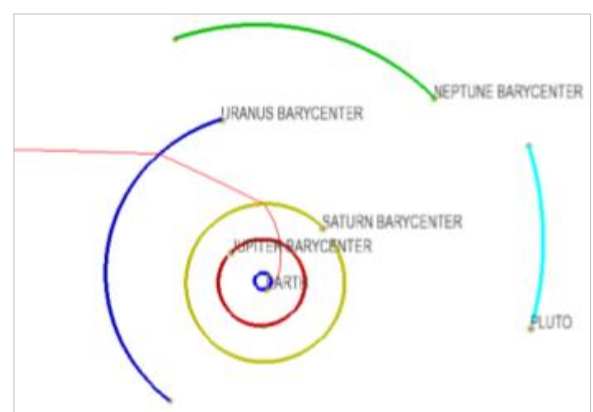

Figure 11. Powered Saturn-Uranus trajectory profile.

The third and final mission profile incorporated a Saturn-Uranus flyby trajectory (Fig. 11), including powered assists at both planetary bodies when possible. To compete with the powered Jupiter flyby, each powered assist would need to provide a $\Delta \mathrm{V}$ of $4 \mathrm{~km} / \mathrm{s}(13,100 \mathrm{ft} / \mathrm{s})$. Unfortunately, after accounting for the additional mass required to add a second kick stage, the entire payload (Interstellar Probe plus two sizeable kick stages) resulted in a mass too heavy for the selected SLS Block 1B architecture. Theoretically, a SLS Block 2B architecture could be assumed but this upgrade will likely not be implemented until much further down the road.

A final analysis was conducted to determine a new total trip time to $250 \mathrm{AU}$ if the mass of the spacecraft and payload attach fitting (PAF) were reduced by half to a combined total of $415 \mathrm{~kg}(915 \mathrm{lb}$ ). By doing so it was found that, for the case with a powered JGA of $4 \mathrm{~km} / \mathrm{s}$, the total trip time could be reduced by approximately 2-3 years. The follow-on Interstellar Probe study discussed subsequently herein also assumes a spacecraft mass close to the 415 $\mathrm{kg}(915 \mathrm{lb})$, at $380 \mathrm{~kg}(838 \mathrm{lb})$ in addition to other adjustments to the GR\&A.

\section{Current Study Analysis}

Following its first workshop which concluded in mid-September 2014, by mid-January 2015 the KISS at Caltech will have concluded its second and final workshop in order to formalize an approach for progressing the proposed Interstellar Probe mission into the next phase of development. The goals of the workshop are listed below followed by the high-level GR\&A for the current study (Table 3).

\section{KISS Workshop Goals: 5}

1) Articulate key scientific questions.

2) Identify near-term science exploration goals.

3) Derive mission objectives and preliminary design concepts that can be realized in the next two decades.

4) Derive flight system and science measurement requirement(s).

5) Assess key mission, system and operations technology drivers.

6) Develop a technology maturation plan that will be proposed to KISS and the Jet Propulsion Laboratory (JPL) for follow-on funding.

Table 3. Ground rules and assumptions for the study discussed herein.

\begin{tabular}{|l|l|}
\hline \multicolumn{1}{|c|}{ Item } & \multicolumn{1}{c|}{ Description } \\
\hline Mission Performance & $100+$ AU in 10 years \\
\hline Launch Window & $2025-2035$ \\
\hline Launch Vehicle & SLS Block 1B + EUS + 8.4 m PLF \\
\hline Spacecraft Mass* & $380 \mathrm{~kg}\left(838 \mathrm{lb}_{\mathrm{m}}\right)$ \\
\hline Spacecraft Heat Shield Mass & $\dagger$ \\
\hline Spacecraft Power & $300 \mathrm{~kg}(661 \mathrm{lb} \mathrm{m})$ \\
\hline
\end{tabular}

* Mass includes all components except onboard low-thrust APS.

${ }^{\dagger}$ Mass scaled from that of Solar Probe Plus heat shield. 


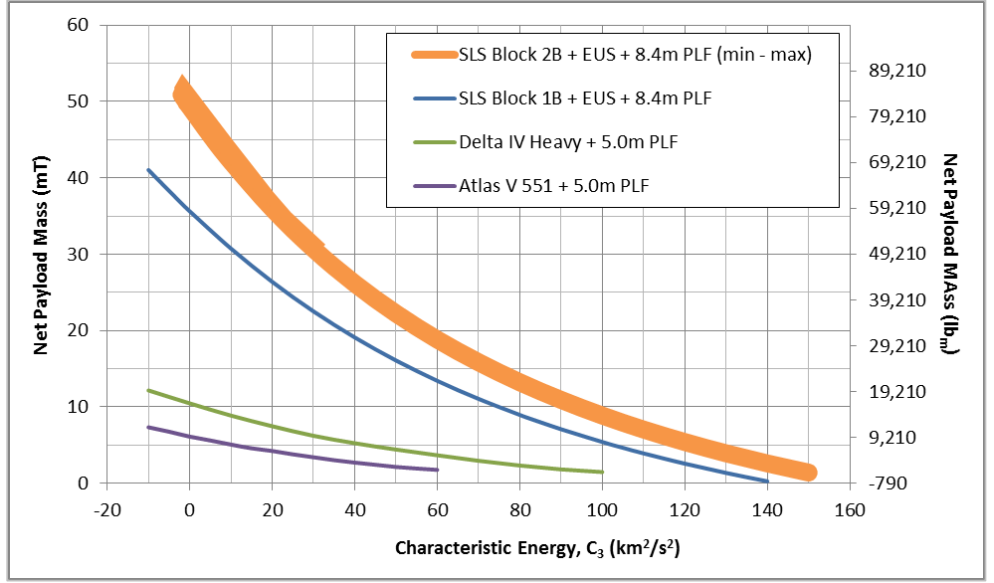

Figure 13. Large launch vehicle ( $L L V) C_{3}$ capabilities. ${ }^{4,6}$

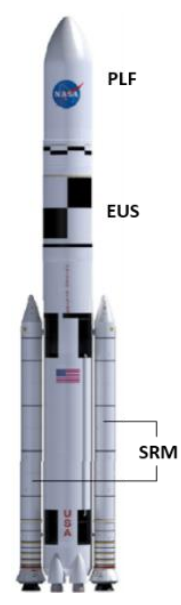

Figure 12. SLS Block 1B with EUS and $8.4 \mathrm{~m} \mathrm{PLF}$. (Image credit: The Boeing Company)

The GR\&A for the current study (Table 3) have been modified from those used in the initial study (Table 2). The overall mission timeline desired was compressed from 30 years to 10 years, which could allow for some of those involved to hopefully one day see the fruits of their labor. Because the launch vehicle industry launch windows tend to be fluidic in nature, the window was shifted so that 2025 is now the earliest potential launch year and spans 10 years to 2035. The same SLS Block 1B vehicle architecture was assumed except with an $8.4 \mathrm{~m}$ (27.6 ft) PLF (Fig. 12) instead of $5.0 \mathrm{~m}$ (16.4 ft) PLF as this was the only Block 1B architecture with a publicly available $\mathrm{C}_{3}$ curve (Fig, 13). ${ }^{4}$ Note that the PAF is bookkept within the net payload mass. As directed by JPL, the total spacecraft mass was assumed to be $380 \mathrm{~kg}(838 \mathrm{lbm})$, which included all components except for the onboard low-thrust APS. It was assumed that $450 \mathrm{~W}$ of power would be available onboard the spacecraft supplied by an Enhanced Multi-Mission Radioisotope Thermoelectric Generator (eMMRTG) ${ }^{7}$ as this technology was considered to be potentially available within the project development timeline given a push to develop it.

It was assumed that a protective heat shield would be attached to the spacecraft, particularly for an impulsive Oberth maneuver performed very close to the sun, approximately 11 solar radii or 0.05 AU above the surface. Its mass was derived by scaling the heat shield currently being designed for NASA's Solar Probe Plus mission by the Johns Hopkins University (JHU) Applied Physics Laboratory (APL) to a size adequate for this mission. ${ }^{8}$

\section{A. Advanced Propulsion Systems}

Several low-thrust APS technologies were traded for each of the trajectory profiles considered, including a MaSMi Hall thruster, solar sails and an E-Sail propulsion system. In addition to the spacecraft having some kind of onboard lowthrust APS stage, the required quantity and size of aft-attached, series-burn SRM kick stages for various impulsive maneuvers was also assessed. The MaSMi hall thruster, would be powered by the onboard eMMRTG outputting $450 \mathrm{~W}$ of power; and, it was assumed, to be capable of 50,000 hours of maximum lifetime and exerting $19 \mathrm{mN}\left(0.004 \mathrm{lb}_{\mathrm{f}}\right)$ of thrust with an $I_{\mathrm{sp}}$ of 1,870 seconds. The solar sail and E-Sail propulsion system GR\&A are outlined below in Tables 4 and 5 , respectively.

Table 4. Solar sail propulsion system GR\&A.

\begin{tabular}{|l|c|c|}
\hline \multicolumn{1}{|c|}{ Item } & \multicolumn{2}{c|}{ Description } \\
\hline Reflectivity & \multicolumn{2}{|c|}{0.91} \\
\hline Minimum Thickness & \multicolumn{2}{|c|}{$2.0 \mu \mathrm{m}$} \\
\hline Maximum Size (per side) & \multicolumn{2}{|c|}{$200 \mathrm{~m}(656 \mathrm{ft})$} \\
\hline Sail Material & \multicolumn{2}{|c|}{$\mathrm{CP} 1$} \\
\hline Aerial Density * & $3 \mathrm{~g} / \mathrm{m}^{2}$ & $10 \mathrm{~g} / \mathrm{m}^{2}$ \\
\hline Characteristic Acceleration & $0.426 \mathrm{~mm} / \mathrm{s}^{2}$ & $0.664 \mathrm{~mm} / \mathrm{s}^{2}$ \\
\hline System Mass & $120 \mathrm{~kg}\left(265 \mathrm{lb} \mathrm{m}_{2}\right.$ & $400 \mathrm{~kg}(882 \mathrm{lb})$ \\
\hline
\end{tabular}

* Assumes an advancement in technology. Current technology is approximately $25 \mathrm{~g} / \mathrm{m}^{2}$.

6

American Institute of Aeronautics and Astronautics 
Table 5. Electric Sail (E-Sail) propulsion system GR\&A.

\begin{tabular}{|l|c|c|}
\hline \multicolumn{1}{|c|}{ Item } & \multicolumn{2}{c|}{ Description } \\
\hline System Mass & \multicolumn{2}{|c|}{$120 \mathrm{~kg}(265 \mathrm{lb}$ m $)$} \\
\hline Wire Material (Density) & \multicolumn{2}{|c|}{ Aluminum $\left(2,800 \mathrm{~kg} / \mathrm{m}^{3}\right)$} \\
\hline Wire Diameter (Gauge) & $0.127 \mathrm{~mm}(36$ gauge $)$ \\
\hline Characteristic Acceleration & $1 \mathrm{~mm} / \mathrm{s}^{2}$ & $2 \mathrm{~mm} / \mathrm{s}^{2}$ \\
\hline Tether Quantity & 10 & 20 \\
\hline Individual Tether Length & $20 \mathrm{~km}(12.4 \mathrm{mi})$ & $20 \mathrm{~km}(12.4 \mathrm{mi})$ \\
\hline
\end{tabular}

\section{B. Trajectory Profile Analysis}

Two trajectory profiles were considered for this study: (1) an escape trajectory using a JGA maneuver $(E-J u)$ and (2) an escape trajectory first performing a JGA maneuver followed by a sun dive via an impulsive Oberth maneuver and Saturn gravity assist maneuver $(E-J u-S u-S a)$. Both trajectory profiles are depicted in Fig. 14 below and are separated based on the type of low-thrust APS employed.
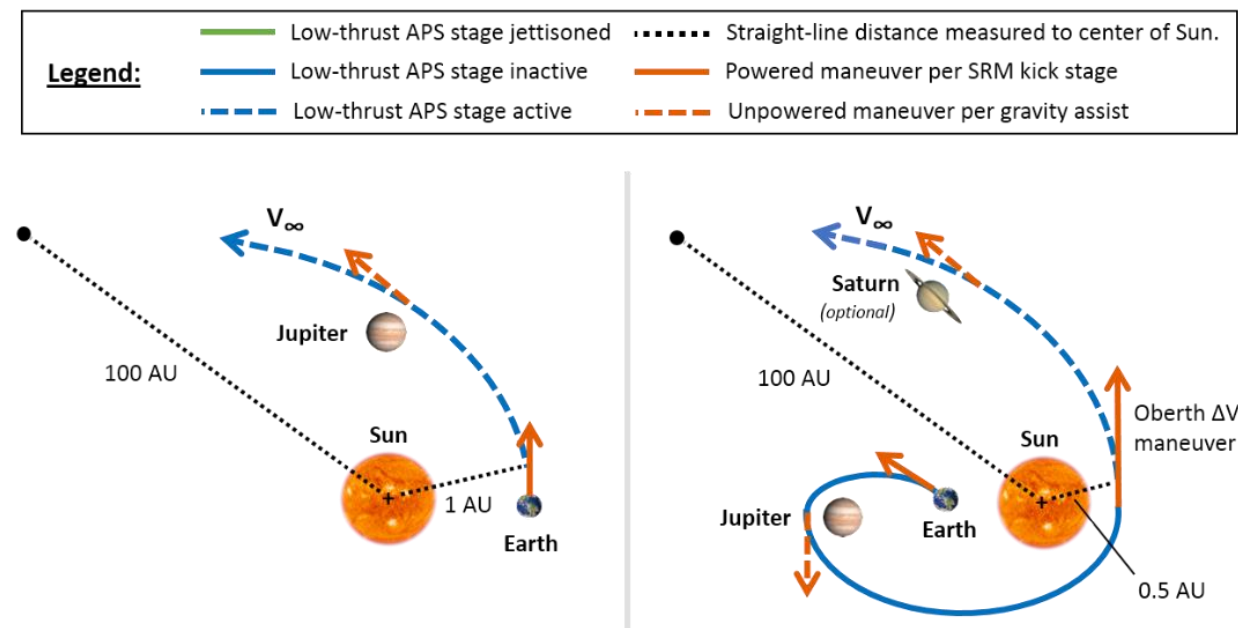

(1a)

(2a)

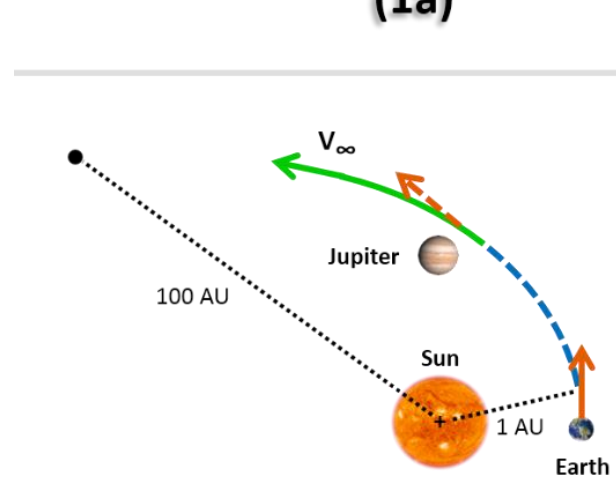

(1b)

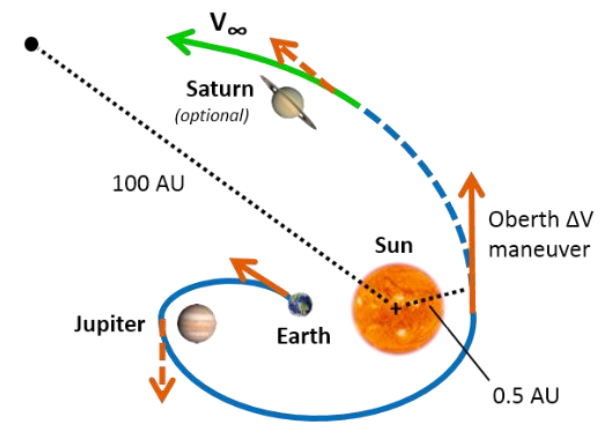

(2b)

Figure 14. Mission trajectory profile options considered: a) trajectories apply to MaSMi Hall thruster and E-Sail systems and b) trajectories apply to solar sail system. 
The first trajectory profile option is similar to the one shown in Fig. 10 except for the lower spacecraft mass, shorter target distance and addition of a low-thrust APS stage. The trajectory relies more heavily on the SLS $\mathrm{C}_{3}$ capability (Fig. 13) which sets the spacecraft's initial velocity prior to earth departure. At departure, the SLS (with an additional SRM kick stage) delivers the spacecraft on an Earth-escape trajectory. Once outside Earth's sphere of influence, the spacecraft deploys and activates its low-thrust APS. For the MaSMi Hall thruster case, the thruster is operated until the assumed 50,000-hour lifetime is reached. If employing a solar sail, the sail is jettisoned prior to the Jupiter gravity assist. The E-Sail option assumes operation until it reaches a point of diminishing return, estimated to occur at about $20 \mathrm{AU}$.

As the spacecraft approaches Jupiter, it performs a gravity assist with a minimum flyby distance of 4.89 Jupiter radii. For this analysis, the orbit of Jupiter is assumed to be circular at 5.203 AU. Figures 15 and 16 illustrate the effect of each low-thrust APS type on the total trip time to the termination shock and heliopause at $100 \mathrm{AU}$. Two E-Sail data points are plotted in Fig. 15 denoted by $\bigcirc$, which corresponds to an E-Sail characteristic acceleration of $2 \mathrm{~mm} / \mathrm{s}^{2}$, and $\square$, which corresponds to a $1 \mathrm{~mm} / \mathrm{s}^{2}$ characteristic acceleration.

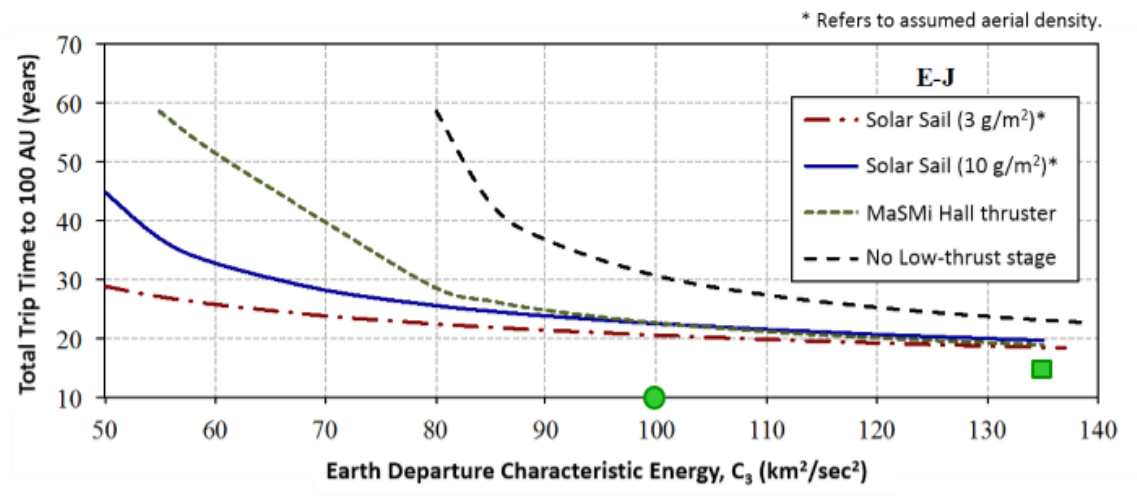

Figure 15. Low-thrust APS analysis for $E$-Ju trajectory profile.

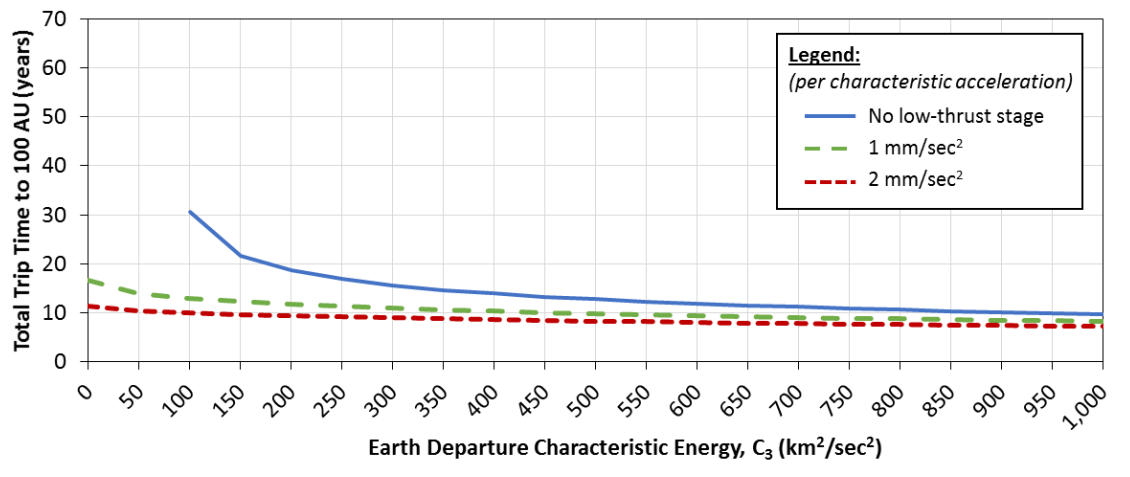

Figure 16. E-Sail propulsion system analysis for $E-J u$ trajectory profile.

Similar to the first trajectory option, the second trajectory begins with an Earth-departure performed by the SLS and an additional SRM kick stage. With the low-thrust APS yet to be activated, the spacecraft performs a Jupiter flyby, which occurs at a minimum passage distance of 18.72 Jupiter radii, in order to reduce its heliocentric speed such that the resulting perihelion is 11 solar radii $(\sim 0.05 \mathrm{AU})$. At perihelion, about 2.97 years into the mission, another SRM kick stage performs the final high-thrust maneuver. Approaching this close to the sun requires that the spacecraft's heat shield withstand temperatures upwards of $2,500{ }^{\circ} \mathrm{F}$ in addition to "blasts of radiation and energized dust levels." ${ }^{8}$ The heat shield, along with the SRM, is jettisoned when the radial distance from the sun is 0.5 AU. This is also where the low-thrust APS is initiated. Similar to the first trajectory option, the MaSMi Hall thruster operates for 50,000 hours, the solar sail is dropped just prior to the next planetary flyby (in this case Saturn), and the E-Sail option is employed until the thrust has a negligible effect. At Saturn, which in this study is assumed to have a circular orbit at $9.583 \mathrm{AU}$, a final gravity assist is performed with a minimal flyby distance of 2.67 planetary radii. Table 6 describes the SRM kick stages chosen for this particular study for various low-thrust APS masses. 
Table 6. SRM kick stages chosen for the $E-J u-S u-S a$ trajectory option.

\begin{tabular}{|l|c|c|l|}
\hline $\begin{array}{c}\text { Low-thrust APS } \\
\text { Stage Mass }\end{array}$ & $\begin{array}{c}\text { Impulsive Burn 1 } \\
\text { (Earth departure) }\end{array}$ & $\begin{array}{c}\text { Impulsive Burn 2 } \\
\text { (Perihelion) }\end{array}$ & \multicolumn{1}{|c|}{ Notes } \\
\hline $0 \mathrm{~kg}\left(0 \mathrm{lb} \mathrm{b}_{\mathrm{m}}\right)$ & Star 63D & Star 48V & Star 63D - 20\% of propellant offloaded. \\
\hline $120 \mathrm{~kg}\left(265 \mathrm{lb} \mathrm{b}_{\mathrm{m}}\right)$ & Star 63F & Star 48V & Star 48V - 5\% of propellant offloaded. \\
\hline $400 \mathrm{~kg}\left(882 \mathrm{lb}_{\mathrm{m}}\right)$ & Star 63F & Star 48V & Star 48V - 20\% of propellant offloaded. \\
\hline $700 \mathrm{~kg}\left(1,543 \mathrm{lb}_{\mathrm{m}}\right)$ & Star 63D & Star 48V & No propellant offloaded for either SRM. \\
\hline
\end{tabular}

Figure 17 below provides additional insight into the target trajectory for option 2 (Fig. 14) where the $\square$ point corresponds to an E-Sail characteristic acceleration of $1 \mathrm{~mm} / \mathrm{s}^{2}$. Figure 18 describes how the performance capability of the SRM kick stage chosen for the Oberth impulsive $\Delta \mathrm{V}$ maneuver affects the total trip time to $100 \mathrm{AU}$.

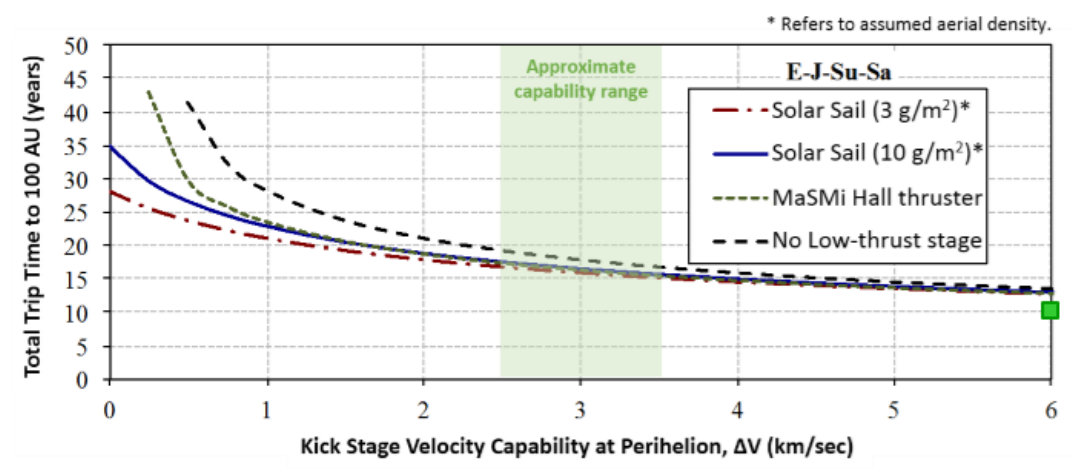

Figure 17. Kick stage analysis for $\mathbf{E}-J u-S u-S a$ trajectory profile.

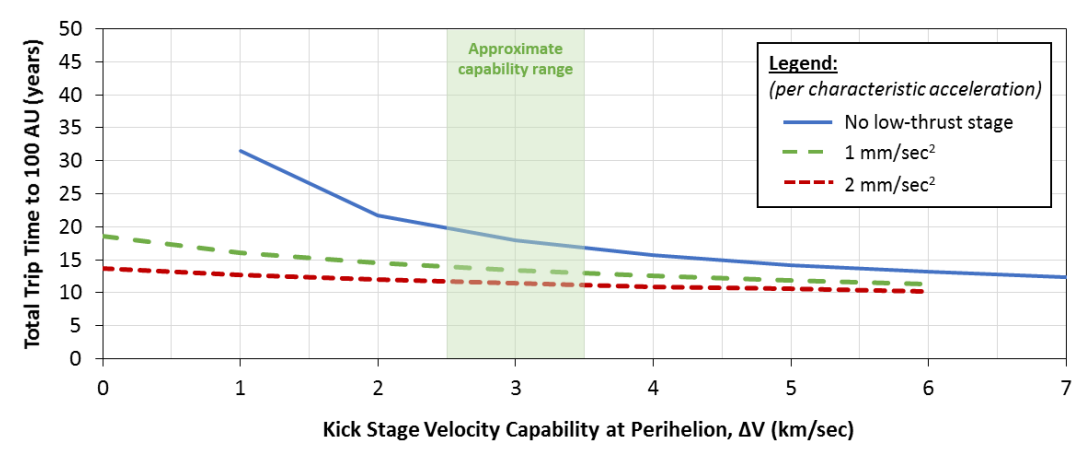

Figure 18. Kick stage analysis for $E-J u-S u-S a$ trajectory profile (E-Sail only).

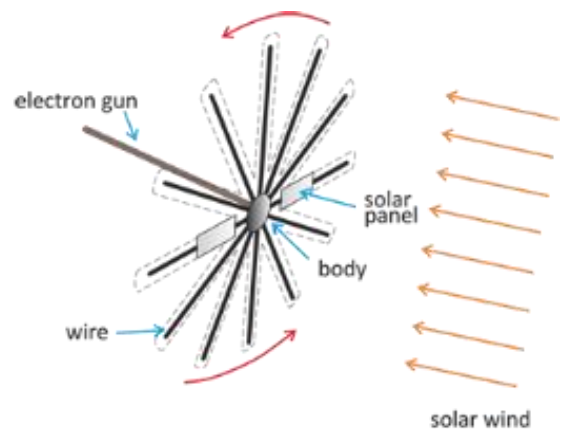

Figure 19. Cartoon schematic of E-Sail propulsion technology.

(Image credit: nextBigFuture.com) 
A quick assessment was conducted to determine what the payload might look like inside of a SLS Block 1B $8.4 \mathrm{~m}$ PLF (Fig. 20). The spacecraft itself was assumed to be volumetrically similar to that of a Voyager 1 or Voyager 2 spacecraft in its stowed configuration. Corresponding to the appropriate low-thrust APS stage mass as shown in Table 6, two SRM kick stages were located below each low-thrust APS stage. The total payload mass was calculated and is quoted above each PLF configuration; it includes the spacecraft bus, low-thrust APS, heat shield and SRM kick stages.
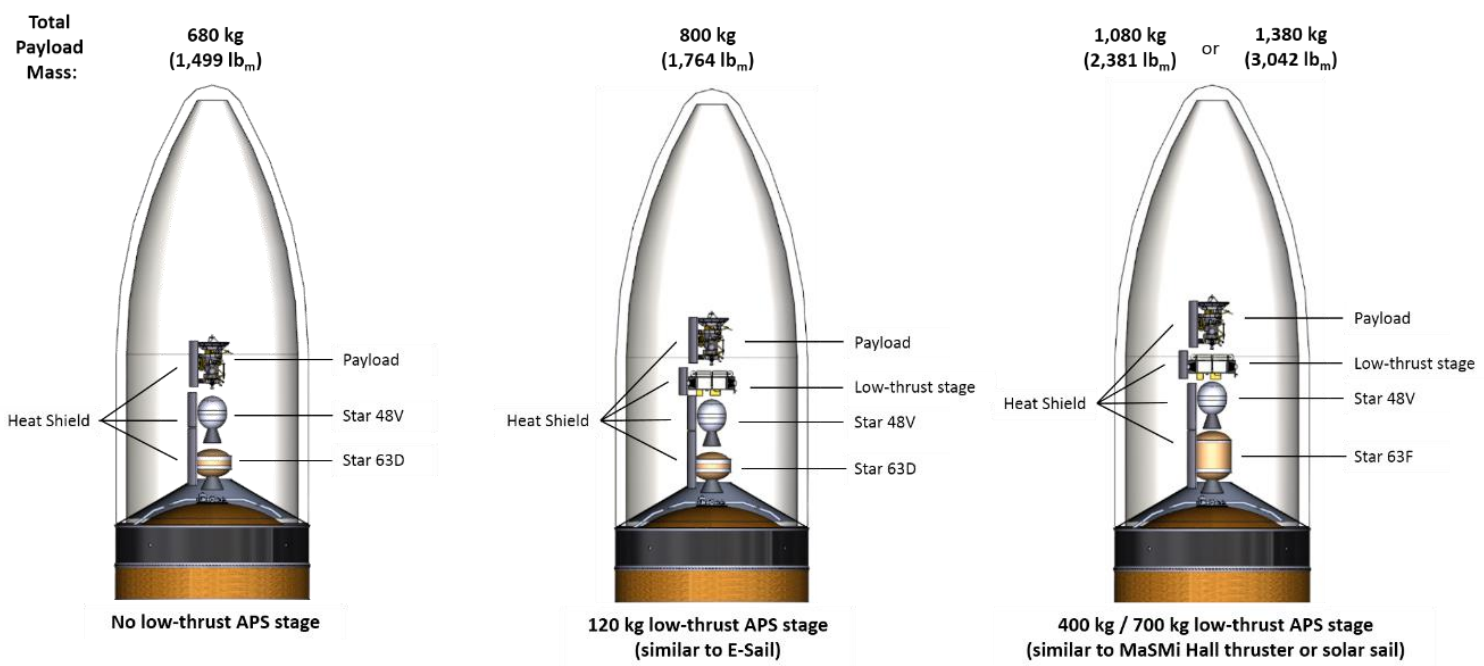

Figure 20. Approximate envelope of payload and SRM kick stages inside SLS 8.4 m PLF per Voyager stow configuration volume.

Figure 21 represents the $E-J u-S u$-Sa trajectory option in a more detailed fashion in order to show the Jupiter flyby and Oberth maneuvers. The figure itself does not include the optional Saturn flyby, which may or may not be available depending on the launch date. Since adjusting the magnitude, timing and direction of the Oberth maneuver may be necessary for targeting a Saturn flyby, variables such as launch window and energy gain imparted by Saturn could vary significantly as well as total trip time.

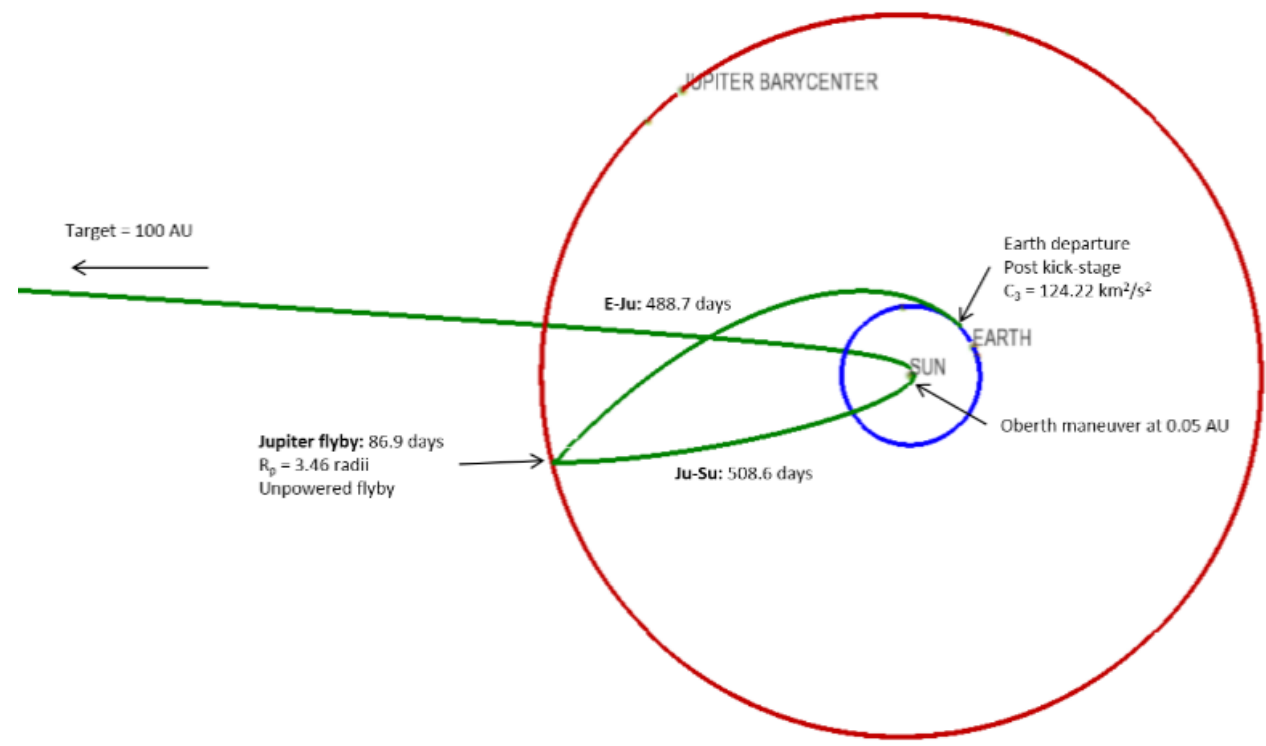

Figure 21. Detailed trajectory for E-Ju-Su-Sa option (Saturn flyby optional). 


\section{Conclusion}

Conceptual analysis concludes that a spacecraft could reach interstellar space within a noticeably shorter amount of time compared to the VIM when employing low-thrust APS stages. In fact, applying an E-Sail low-thrust APS stage results in the lowest total trip time of approximately 11 years for the $E$-Ju-Su-Sa trajectory option. There is also an additional potential mass, and thus time, savings if a SLS Block 1B $5.0 \mathrm{~m}$ PLF is employed rather than the $8.4 \mathrm{~m}$ PLF since there appears to be adequate room to do so. With that said, all low-thrust APS technologies for either trajectory option provide substantial total trip time improvements over the VIM ranging anywhere from 11 - 19 years, assuming a maximum applied $\mathrm{C}_{3}$ capability of $135 \mathrm{~km}^{2} / \mathrm{s}^{2}$ for an $E$-Ju- trajectory profile or an average perihelion kick stage $\Delta \mathrm{V}$ of $3 \mathrm{~km} / \mathrm{s}(9,842 \mathrm{ft} / \mathrm{s})$ for the $E-J u$-Su-Sa trajectory profile. However, for the latter profile, compared to the MaSMi hall thruster and solar sail propulsion systems, having no low-thrust APS stage actually achieves almost the same total trip time especially for perihelion kick stage $\Delta V$ 's greater than $4 \mathrm{~km} / \mathrm{s}(13,123 \mathrm{ft} / \mathrm{s})$.

It is worth noting that there is one concern specifically for the E-Ju-Su-Sa trajectory option and that is the SRMpowered Oberth maneuver performed very close to the sun's surface (11 solar radii or $0.05 \mathrm{AU})$. Keeping in mind that this is a conceptual study, the assumed heat shield technology is stemming from NASA's Solar Probe Plus mission, which is scheduled to occur before the Interstellar Probe mission. It is uncertain as to how the heat shield would cover the SRM kick stage performing the impulsive perihelion burn while not being simultaneously partially destroyed by the kick stage's plume. In other words, if the heat shield incurs damage then it is a concern as to how the rest of the shield would perform for the duration of the trajectory where the spacecraft is still very close to the sun.

\section{Acknowledgements}

The authors acknowledge ideas and advice from the participants in the Science and Enabling Technologies to Explore the Interstellar Medium workshops organized by the W.M. Keck Institute for Space Studies.

\section{References}

1 “The Mission Overview: Interstellar Mission," Jet Propulsion Laboratory (JPL), California Institute of Technology, URL: http://voyager.jpl.nasa.gov/mission/interstellar.html [cited 30 December 2014].

2 "The Mission Overview: Fast Facts," Jet Propulsion Laboratory (JPL), California Institute of Technology, URL: http://voyager.jpl.nasa.gov/mission/fastfacts.html [cited 30 December 2014].

3 "Science and Enabling Technologies to Explore the Interstellar Medium," Keck Institute for Space Studies (KISS) at California Institute of Technology, URL: http://www.kiss.caltech.edu/study/science.html [cited 30 December 2014].

4 “Space Launch System (SLS) Program Mission Planner's Guide (MPG) Executive Overview (Version 1)," SLS-MNL-201, NASA MSFC, August 22, 2014.

5 "Science and Enabling Technologies to Explore the Interstellar Medium II," Keck Institute for Space Studies (KISS) at California Institute of Technology, URL: http://www.kiss.caltech.edu/study/scienceII/index.html [cited 2 January 2015].

6 McNutt, Jr., R. L., "Enabling Interstellar Probe with Space Launch System (SLS)," IAC-14-D.4.4.2, 65 ${ }^{\text {th }}$ International Astronautical Congress (IAC), Johns Hopkins University (JHU) Advanced Physics Laboratory (APL) and The Boeing Company, page 6, 2014.

7 "Enhanced Multi-Mission Radioisotope Thermoelectric Generator (eMMRTG) Concept," NASA, URL: https://solarsystem.nasa.gov/rps/docs/eMMRTG_onepager_LPSC20140317.pdf [cited 2 January 2015].

8 "Mission Overview," NASA and JHU APL, URL: http://solarprobe.jhuapl.edu/mission/ [cited 1 January 2015].

${ }^{9}$ Quarta, A. A., Mengali, G., "Electric Sail Mission Analysis for Outer Solar System Exploration, University of Pisa, Italy. 